\title{
'They won't do any harm and might do some good': time to think again on the use of antidepressants?
}

\author{
Hugh Middleton and Joanna Moncrieff
}

\author{
ABSTRACT \\ Despite NICE guidance that should have limited it, \\ antidepressant prescribing continues to increase. \\ Research evidence suggests that much if not all of the \\ observed efficacy should be attributed to complex \\ non-specific effects rather than 'restoration of \\ disturbed brain chemistry'. According to this view the \\ uncertain benefits of antidepressants are unlikely to \\ outweigh the risks, suggesting the need to explore \\ other approaches to treatment. \\ Keywords \\ antidepressant; depression; efficacy; placebo; sick role; \\ side effects.
}

\section{INTRODUCTION}

The April 2010 edition of BJGP carried several articles referring to practitioners' concerns about high rates of prescribing psychotropic medication in primary care..$^{1-3}$ These concerns are probably most widely felt in relation to serotonin-selective reuptake inhibitors (SSRIs) and other so-called antidepressants, prescription of which has increased from less than 10 million in 1991 to almost 40 million in 2009. There is no evidence that the 2004 NICE recommendation to limit first-line use to moderate to severe depression has had any impact (Figure 1). ${ }^{4-6}$ Furthermore these levels of prescribing have not been associated with a reduction in the related burden of care, with the number of people claiming incapacity benefit for a mental or behavioural disorder rising steadily from under 750000 in 2000 to nearly a million in 2008 (Figure 2). ${ }^{\text {? }}$

The association between rising levels of drug treatment and increasing disability may not be surprising in the light of recent research on antidepressants which suggests they may not be superior to placebo, certainly for the majority of patients with milder degrees of depression. ${ }^{8,9}$ Even for those with more severe depression (Hamilton Rating Scale for Depression [HRSD] score >24), doubts remain about whether the effects observed are clinically meaningful. ${ }^{10}$ In any event, in a recent survey, $71 \%$ of 'depressed' treatment-seeking outpatients had a HDRS score <22. ${ }^{11}$ Every day thousands of prescriptions for antidepressants are written without any likelihood that their effects will differ from placebo, with considerable implications for avoidable harm and opportunities for financial saving.

These reflections suggest we may need to reexamine some assumptions about depression and its treatment, especially the widely-held aphorism that drug treatment 'won't do any harm and might do some good'. It is timely to think more laterally about why we prescribe antidepressants, what is achieved (and risked) when we do, and how the needs of patients with depression might be better met.

\section{THE EVIDENCE ON ANTIDEPRESSANT EFFECTS}

The use of 'antidepressants' is based on the widely- 


\section{How this fits in}

Steadily increasing rates of psychotropic and antidepressant prescribing are associated with growing concern about the quality of evidence upon which this is based. The notion of depression as 'a disturbance of brain chemistry which can be corrected by the use of antidepressants' is seriously flawed. It may be more accurate to consider their benefits, when present, attributable to complex nonspecific effects not unlike a placebo. This change in perspective has implications for practice which should include a more considered approach to the balance between potentially beneficial and undoubtedly harmful consequences of prescription.

Figure 1. Rates of antidepressant prescribing in England: 1991 to 2009.

Figure 2. Rates of Incapacity Benefit Claim for Mental and Behavioural Disorder 2000 to 2009.
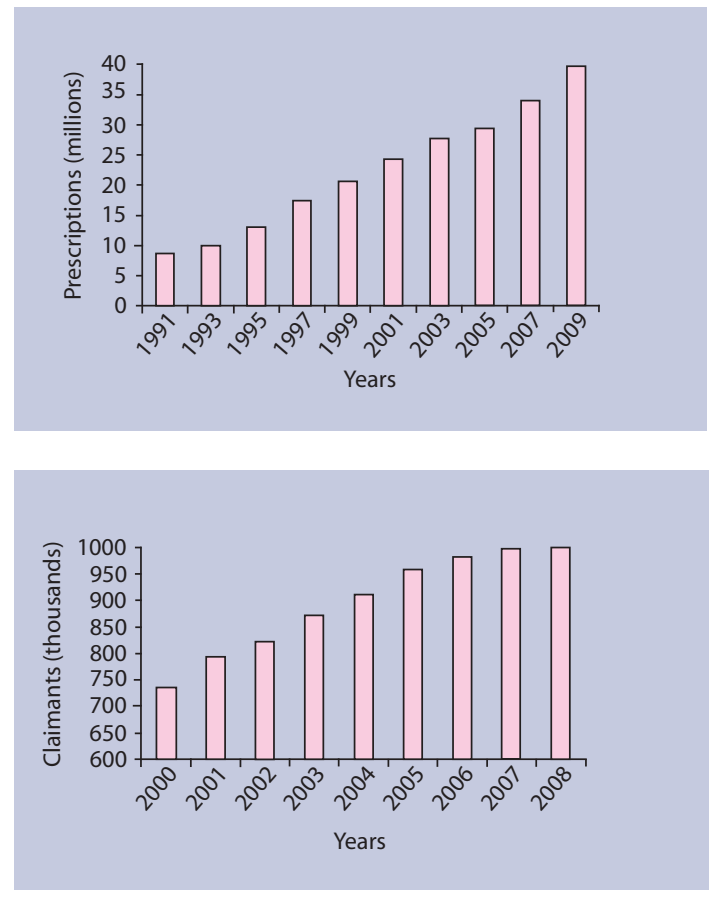

held and promulgated view that depression and related conditions can be usefully understood as potentially remediable disturbances of brain chemistry. This is not an unquestioned position, however, and evidence that there are specific abnormalities of serotonin or noradrenaline in people diagnosed with depression remains inconclusive. ${ }^{10}$ Even if depression were to be associated with a specific biochemical disturbance, we have no current grounds to claim that antidepressant drugs act by reversing this abnormality. ${ }^{12}$ Apart from placebo effects, antidepressants exert psychoactive effects that may reveal their identity to people taking part in placebo controlled trials, thus infringing the double blind design. Moreover, the psychoactive effects of antidepressants, such as the sedative effects of tricyclics and the possible emotional dampening effects of SSRIs, ${ }^{13}$ may themselves impact on outcome measures. The fact that psychoactive substances not normally considered to be antidepressants, including antipsychotics, stimulants, and benzodiazepines have similar effects to antidepressants in some trials, and that antidepressants themselves come from diverse chemical classes, supports the idea that it is the psychoactive and placebo effects of antidepressants, not their supposed effect on an underlying brain disorder, that accounts for the small differences observed between drugs and placebos in randomised controlled trials. ${ }^{12}$

Despite this lack of evidence, we acknowledge that a substantial body of opinion continues to maintain that depression is a reversible, or partially reversible brain disease that can usefully be treated with drugs. The argument that this can best be understood as an 'ideology' deliberately maintained by those with a commercial interest in doing so is presented elsewhere,$^{14}$ although it is indicative of changes in this ideology that quetiapine is now being marketed in the US as a treatment for depression and bipolar disorder as well as its original indication, schizophrenia. ${ }^{15}$ Unsurprisingly there have been criticisms and reanalyses of data which show little difference between antidepressants and placebo such as Kirsch et al's study, ${ }^{8}$ but they have yet to offer substantially different conclusions. One such is a recent review ${ }^{16}$ in which the use of a different and less well accepted statistical technique (weighting by sample size rather than the inverse variance) resulted in a minimally larger (around half a point on the HRSD) points estimate of that difference. We invite readers to form their own opinions. We are also aware that questioning this view does not tell us how to respond to those who seek help with their distress. What are the practical implications of abandoning the assumption that 'Depression is a disturbance of brain chemistry which can be corrected by the use of antidepressants'?

\section{IMPLICATIONS}

The implications of departing from an ideology which argues 'Depression is a disturbance of brain chemistry which can be corrected by the use of antidepressants' are quite profound. For instance, there is no obligation to prescribe. Although the temporary use of sedatives might be justified for agitation or insomnia, if antidepressants are not considered to have a specific effect on an underlying disorder, then it is not negligent not to prescribe and to try other approaches instead. Antidepressants cause a range of unpleasant side effects, including sexual side effects, drowsiness, emotional flattening, and restlessness and they may occasionally give rise to increased suicidal impulses. These all assume greater significance if the benefits of treatment are uncertain.

Whether or not antidepressants exert any specific effect in depression, we know that the majority of the improvement seen in randomised controlled trials is replicated in the placebo group. ${ }^{17}$ Although some of 
this improvement is likely to represent the natural course of the disorder, expectancy or placebo effects are also likely to play an important role. Where the placebo effect has been studied in detail it appears to involve three core components; acknowledgment of the patient's difficulties by paying attention to the problem, a credible therapeutic ritual, and the quality of the patient-practitioner relationship. ${ }^{18}$ Antidepressant medication may exert an effect not because of its pharmacology, but because a prescription provides at least two of these. The problem is acknowledged (it is an illness) and a credible (antidepressant) treatment is provided.

\section{THE SYMBOLISM OF TREATMENT}

Therefore, it may be more productive to view the presentation of emotional distress in a medical setting as a situation to be understood and addressed, rather than an illness awaiting treatment. This allows practitioners to reflect on how the elements of the placebo effect may be utilised without recourse to unnecessary drug treatment. Acknowledgement of the problem and a credible therapeutic ritual may be achieved through counselling or cognitive behaviour therapy (CBT), for example, but identifying the source of patients' difficulties and their reasons for seeking medical advice remain important, even before therapy is instituted. The patient, not the doctor, is the expert in this situation, and the role of the doctor is to help and support the patient in identifying the nature of their problems and how to address them.

Some patients will want their distress to be acknowledged within a medical framework, and may respond to the acknowledgment and hope a prescription can provide. In the long term, however, the message that is symbolised by medication, may be harmful. Symbolically, medication suggests that the problem is within the brain, and that wellbeing is dependent upon maintaining 'chemical balance' by artificial means, a message which may encourage people to view themselves as flawed and vulnerable, and may explain the poor outcomes of treated depression in naturalistic studies. ${ }^{19}$ Probably most harm is done when the view develops, that successful 'treatment' depends upon finding the right antidepressant, and the patient works through a series of different medications. Many patients do not necessarily seek drug treatment, however, and wait to be guided by the practitioner.

\section{CHANGING PRACTICE}

The Improved Access to Psychological Therapies (IAPT) programme ${ }^{20}$ is now under way and is beginning to provide the practitioner with alternatives to drug treatment. We have yet to see whether it will be used instead or as well as antidepressants but there is no evidence of that to date (Figure 1). If this is to happen it will depend upon whether doctors continue to allow themselves to be persuaded that antidepressants 'can't do any harm and might do some good', or begin to recognise that they are 'unlikely to do any good and may do some harm'.

\section{Competing interests}

The authors have stated that there are none.

Discuss this article

Contribute and read comments about this article on the Discussion Forum: http://www.rcgp.org.uk/bjgp-discuss

\section{REFERENCES}

1. Dickinson R, Knapp P, House AO, et al. Long-term prescribing of antidepressants in the older population: a qualitative study. Br J Gen Pract 2010; 60(573): e144-155.

2. Lasserre A, Younès N, Blanchon T, et al. Psychotropic drug use among older people in general practice: discrepancies between opinion and practice. Br J Gen Pract 2010; 60(573): e156-162.

3. Siriwardena AN. Why do GPs prescribe psychotropic drugs when they would rather provide alternative psychological interventions? $\mathrm{Br}$ $J$ Gen Pract 2010; 60(573): 241-242.

4. National Institute for Clinical Excellence (NICE). The management of depression in primary and secondary care. London: National Institute for Clinical Excellence, 2004.

5. Double D. The limits of psychiatry. BMJ 2002; 324(7342): 900-904.

6. NHS Information Centre. Prescriptions. http://www.ic.nhs.uk/statistics-and-data-collections/primarycare/prescriptions (accessed 22 Nov 2010).

7. Department for Work and Pensions. Department for Work and Pensions statistical tabulations. http://83.244.183.180/100pc/tabtool.html (accessed 22 Nov 2010).

8. Kirsch I, Deacon BJ, Huedo-Medina TB, et al. Initial severity and antidepressant benefits: a meta-analysis of data submitted to the Food and Drug Administration. PLoS Med 2008; 5: e45. doi:10.1371/journal.pmed.0050045

9. Fournier JC, DuRubeis RJ, Hollon S, et al. Antidepressant drug effects and depression severity: a patient-level meta-analysis. JAMA 2010; 303(1): 47-53.

10. Moncrieff J, Cohen D. How do psychiatric drugs work? BMJ 2009; 338: 1535-1537.

11. Zimmerman M, Posternak MA, Chelminski I. Symptom severity and exclusion from antidepressant efficacy trials. J Clin Psychopharmacol 2002; 22: 610-614.

12. Moncrieff J, Cohen D. Do antidepressants cure or create abnormal brain states? PLoS Med 2006; 3(7): e240.

13. Price J, Cole V, Goodwin GM. Emotional side-effects of selective serotonin reuptake inhibitors: qualitative study. Br J Psychiat 2009; 195(3): 211-217.

14. Moncrieff J. The myth of the chemical cure: a critique of psychiatric drug treatment. Basingstoke, Hampshire: Palgrave Macmillan, 2008.

15. Seroquel XR. (quetiapine fumerate) http:/www.seroquelxr.com (accessed 26 Nov 2010).

16. Fountoulakis KN, Moller HJ. Efficacy of antidepressants: a re-analysis and re-interpretation of the Kirsch data. Int J Neuropsychopharmacol 2010; Aug 27: 1-8. [Epub ahead of print].

17. Kirsch I, Moore TJ, Scoboria A, Nicholls SS. The emperor's new drugs: an analysis of antidepressant medication data submitted to the US Food and Drug Administration. Prevention and Treatment 2002; 5: alphachoices.com/repository/assets/pdf/EmperorsNewDrugs.pdf. (accessed 22 Nov 2010).

18. Kaptchuk TJ, Kelley JM, Conboy LA, et al. Components of placebo effect: randomised controlled trial in patients with irritable bowel syndrome. BMJ 2008; 336(7651): 999-1003.

19. Goldberg D, Privett M, Ustun B, et al. The effects of detection and treatment on the outcome of major depression in primary care: a naturalistic study in 15 cities. Br J Gen Pract 1998; 48(437): $1840-1844$.

20. NHS. Improving access to psychological therapies. http://www.iapt.nhs.uk/ (accessed 11 Nov 2010). 